\title{
Decoding Galactic Merger Histories
}

\author{
Eric F. Bell ${ }^{1, *}$ (D), Antonela Monachesi ${ }^{2}$, Richard D'Souza ${ }^{1,3}$, Benjamin Harmsen ${ }^{1}$, \\ Roelof S. de Jong ${ }^{4}$, David Radburn-Smith ${ }^{5}$, Jeremy Bailin ${ }^{6}$ (i) and Benne W. Holwerda ${ }^{7}$ \\ 1 Department of Astronomy, University of Michigan, Ann Arbor, MI 48109, USA; radsouza@umich.edu (R.D.); \\ benharms@umich.edu (B.H.) \\ 2 Departamento de Física y Astronomía, Universidad de La Serena, La Serena 1720236, Chile; \\ amonachesi@userena.cl \\ 3 Vatican Observatory, Piazza Sabatini, 4B/5, 00041 Albano Laziale RM, Italy \\ 4 Leibniz-Institut für Astrophysik Potsdam (AIP), 14482 Potsdam, Germany; rdejong@iap.de \\ 5 Department of Astronomy, University of Washington, Seattle, WA 98195, USA; d.radburnsmith@gmail.com \\ 6 Department of Physics and Astronomy, University of Alabama, Tuscaloosa, AL 35487, USA; jbailin@ua.edu \\ 7 Department of Physics and Astronomy, University of Louisville, Louisville, KY 40292, USA; \\ benne.holwerda@gmail.com \\ * Correspondence: ericbell@umich.edu; Tel.: +1-734-764-3408
}

Received: 30 June 2017; Accepted: 1 December 2017; Published: 8 December 2017

\begin{abstract}
Galaxy mergers are expected to influence galaxy properties, yet measurements of individual merger histories are lacking. Models predict that merger histories can be measured using stellar halos and that these halos can be quantified using observations of resolved stars along their minor axis. Such observations reveal that Milky Way-mass galaxies have a wide range of stellar halo properties and show a correlation between their stellar halo masses and metallicities. This correlation agrees with merger-driven models where stellar halos are formed by satellite galaxy disruption. In these models, the largest accreted satellite dominates the stellar halo properties. Consequently, the observed diversity in the stellar halos of Milky Way-mass galaxies implies a large range in the masses of their largest merger partners. In particular, the Milky Way's low mass halo implies an unusually quiet merger history. We used these measurements to seek predicted correlations between the bulge and central black hole (BH) mass and the mass of the largest merger partner. We found no significant correlations: while some galaxies with large bulges and BHs have large stellar halos and thus experienced a major or minor merger, half have small stellar halos and never experienced a significant merger event. These results indicate that bulge and BH growth is not solely driven by merger-related processes.
\end{abstract}

Keywords: galaxies: general; galaxies: evolution; galaxies: halos; galaxies: stellar content; galaxies: bulges; galaxies: merger history

\section{Introduction}

The cold dark matter paradigm predicts that the gravitational collapse and merger of dark matter halos is the prime driver of galaxy formation and growth. However, the response of stars and gas to these mergers is complex to model, and predictions of their effects on galaxy bulges, disks, and supermassive black holes are uncertain (e.g., [1,2]). Direct knowledge of the merger histories of galaxies would therefore be valuable in providing direct empirical tests of how a given merger affected a particular galaxy. Sadly, dark matter is, well... dark, and direct measurement of the merger history is impossible. Yet, because the largest dark matter subhalos are predicted to host visible satellite galaxies, we can use these satellites as visible tracers of the growth of the central galaxy's dark matter halo. The tidal disruption of these satellites is predicted to form a diffuse stellar halo (e.g., [3-6]). Consequently, study of these stellar halos gives unique—and perhaps the only available—insight into 
the merger and growth history of actual galaxies, and offers the possibility of understanding what mergers do to galaxies. Here we summarize the findings of [7-10] and give a brief overview of the progress that has been made towards measuring the stellar halos around nearby Milky Way mass galaxies, using them to infer the most prominent event in their merger and accretion histories, and using this knowledge to explore the role of merging in bulge and supermassive black hole growth.

This issue is the most urgent for Milky Way (MW) peers-galaxies with $M_{*} \sim 6 \times 10^{10} M_{\odot}$. In addition to holding most of the stellar mass in the present-day Universe [11], MW peers are diverse (e.g., [12]), spanning from bulgeless star-forming disks like the Milky Way or M101 to elliptical or lenticular galaxies like Centaurus A or the Sombrero galaxy. It is thought that much of this diversity should be driven by merger history (e.g., [1,2]). Galaxy merging plays an important role in the creation of elliptical galaxies (e.g., [13,14]). It is commonly argued that mergers are an important driver of the formation of at least the large "classical" galaxy bulges (e.g., $[1,2,15])$, although other mechanisms such as early bulge formation through chaotic collapse (e.g., [16]), violent disk instabilities (e.g., [17]), or dramatic changes in gas angular momentum [18] have been suggested. Further, given that feedback from supermassive black holes (BHs) may suppress star formation on galactic scales (e.g., $[19,20]$ ) and that merging appears to drive at least some $\mathrm{BH}$ growth [21,22], it is important to test the relationship between $\mathrm{BH}$ mass and merger history directly.

In order to assess what mergers do to galaxies (do mergers grow BHs, bulges, elliptical galaxies?), an independent and robust probe of the merger history of individual galaxies is urgently needed. Stellar halos appear to be just such a probe (see [7-10] for more details).

\section{Stellar Halos Measure Merger History}

Galactic outskirts are predicted to give powerful insight into their merger and accretion histories. Stars in merging galaxies are collisionless, and are torn by tides from their parent galaxy and spread out into a diffuse stellar halo (e.g., [4,6]). Because the stellar mass of satellites is a very strong function of dark matter subhalo mass, these stellar halos are predicted to accrete most of their mass from the few largest, and in most cases the single largest, satellite(s) (e.g., [5,10,23]).

This is illustrated in Figure 1. We use the Illustris hydrodynamical simulation $[10,24]$ to predict the fraction of the accreted mass given by the single-most massive disrupted satellite (frac ${ }_{\text {Dom }}$ on the $x$-axis) and the ratio of the second-most massive to the most massive progenitor (on the $y$-axis) for a sample of simulated galaxies with dark matter halo masses similar to the Milky Way $\left(12.05<\log M_{D M} / M_{\odot}<12.15\right)$. A wide range of growth histories are predicted, from (relatively uncommon) stellar halos built up from multiple smaller accretions (top left) through to halos completely dominated by a single massive accretion (bottom right). The growth history broadly correlates with total accreted mass, where low-mass stellar halos are often built up from many smaller accretions, while typical and large stellar halos are dominated by a single accretion (in agreement with [23]). Most MW peer stellar halos are predicted to have more than half of their total halo mass come from the most massive progenitor, and usually this most massive progenitor is substantially more massive than the second-most massive progenitor. Because the only model ingredients required to robustly predict global stellar halo properties are realistic galaxy metallicity-mass relations and mass functions of accreted satellites $[5,25]$, these predictions are robust and other models $[23,26]$ give very similar results.

This framework makes an important prediction. Since galaxies show a strong relationship between their metallicity and stellar mass [27], and because stellar halos tend to be dominated by the most massive progenitor (see Figure 1 and references [10,23]), the metallicity and mass of accreted stellar halos are predicted to correlate strongly $[8,10,23]$. This offers a clear observational test of the accretion-driven growth of stellar halos, and we will return to it in Figures 2 and 3. 


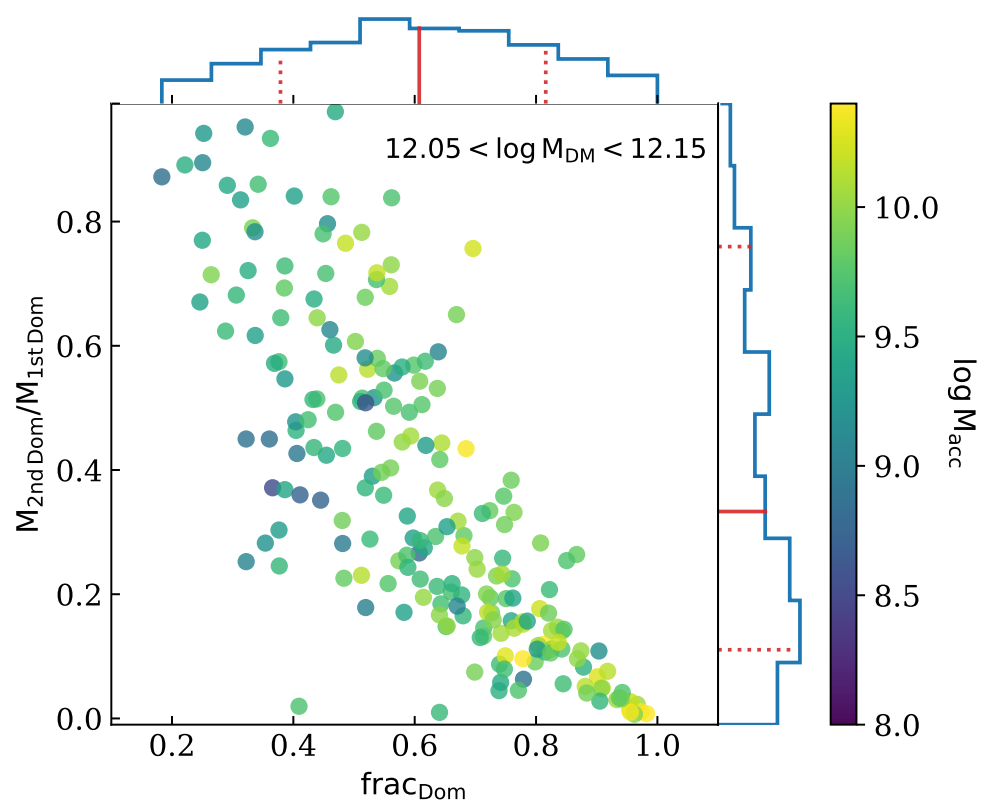

Figure 1. Stellar halos are predicted to have accreted their mass from the few largest, and in many cases the single largest, satellite(s). The $x$-axis shows frac $_{\text {Dom, }}$, the fraction of the accreted mass given by the single-most massive disrupted satellite as predicted by the Illustris hydrodynamical simulation $[10,24]$ for a sample of simulated galaxies with dark matter halo masses similar to the Milky Way $\left(12.05<\log M_{D M} / M_{\odot}<12.15\right)$. The $y$-axis shows the ratio of the second-most massive to the most massive progenitor. Symbols are color-coded by the total accreted mass. Marginalized distributions of each quantity are shown, along with the 16,50, and 84 percentiles of the distributions. The stellar halos of Milky Way (MW) peers are expected to show a wide range of properties, from the less-common halos built up from multiple smaller accretions (top left) through to the more common halos completely dominated by a single massive accretion (bottom right).

While it is not the focus of this contribution, in addition to the accreted stellar halo, there is considerable discussion about the extent to which stars formed in the main galaxy itself will exist at large radius - an in situ stellar halo. Simulators report that the prominence and origin of in situ stars varies considerably as a function of the parameters of simulations (e.g., the parameterization of stellar feedback, [28,29]). At the level of comparing observations with simulated total stellar halo masses, many simulations appear to over-produce in situ stellar halos (e.g., $[8,10])$.

While we await a clearer understanding of in situ stars, we have chosen to focus on the minor axis of galaxies with prominent disks. High-resolution hydrodynamical simulations predict that at minor axis distances $>15 \mathrm{kpc}$ in MW peers, most stars should be accreted [30,31]. Estimates of total accreted mass and median metallicity can be inferred from minor axis measurements [10]. Using these estimates, one can measure the mass of the most massive merger partner (e.g., Figure 1 and reference [10]) and possibly when this merger occurred [10,23]. Consequently, it is important to carefully characterize the diffuse stellar halos-ideally along their minor axes-for a representative sample of galaxies.

The astronomical community is starting to assemble just such a sample. Aside from prominent individual stellar streams (e.g., [32,33]), the characterization of stellar halos has been very challenging owing to their extremely low surface brightnesses $>30 \mathrm{~V}$-band mag/arcsec ${ }^{2}$. Recent deep diffuse light imaging has permitted detection of relatively massive halos (e.g., [34-36]). In parallel, studies of resolved red giant branch (RGB) stars in the outskirts of nearby galaxies reach fainter equivalent surface brightness limits and give estimates of both stellar halo masses and typical metallicities [7,8,37-41]. 

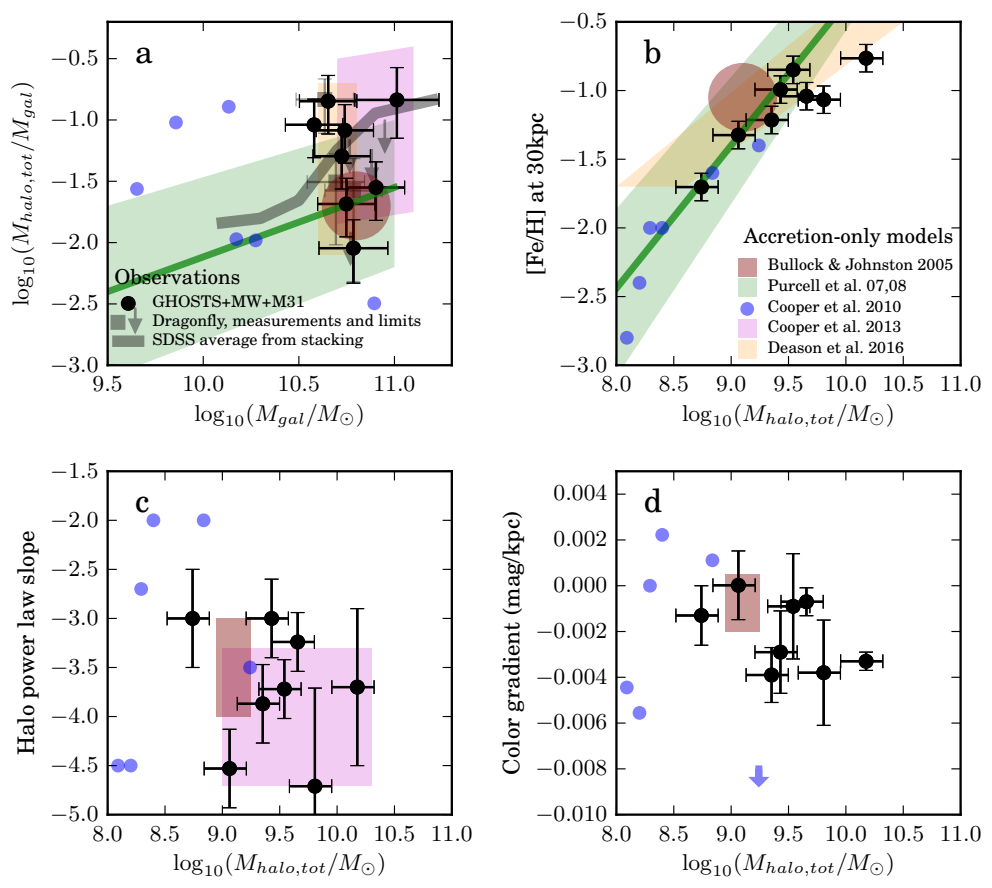

Figure 2. Both resolved star (black; $[7,8,38,39,42-44]$ ) and integrated light studies (gray; [36,45]) reveal a diversity of stellar halo masses, metallicities, density profiles, and metallicity gradients. These observational properties are in good agreement with models in which stellar halos are predominantly formed by the disruption of dwarf satellites (in colors). (a) Ratio of "total" stellar halo mass and total stellar mass, as a function of total stellar mass. Error bars include the uncertainty in extrapolating to "total" stellar halo mass; limits are shown with arrows. (b) Stellar halo metallicity at $30 \mathrm{kpc}$ as a function of "total" stellar halo mass. (c) Inferred 3D minor axis stellar halo density power-law slope in the range $10-40 \mathrm{kpc}$ as a function of "total" stellar halo mass. (d) Stellar halo color gradient (a proxy for metallicity gradient) as a function of "total" stellar halo mass. The observational data are shown in black and grey. Models: brick red area: [4]; light green+line: [5,25]; blue: [6]; magenta: [26]; orange: [23]. Adapted from Figure 16 from [8], reproduced with permission.

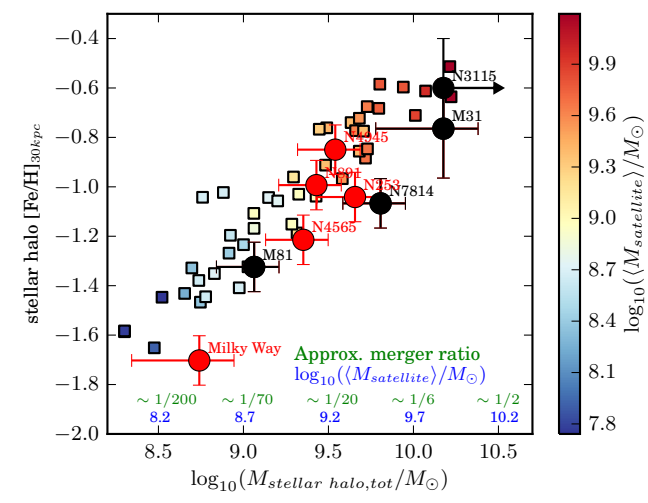

Figure 3. Circles show the observed correlation between stellar halo mass and metallicity. Galaxies with "classical" bulges are shown in black, galaxies with "pseudobulges" are shown in red. Squares show simulated stellar halo masses and metallicities from the accretion-only models of Deason et al. [23]. The squares are color-coded according to the mass weighted mean stellar mass of the contributing satellites to the halo; we indicate the approximate run of mass weighted mean accreted satellite mass in blue and corresponding approximate merger ratios for a main galaxy mass at the time of merger of $\sim 3 \times 10^{10} M_{\odot}$ in green. Adapted from Figure 1 of [9], reproduced with permission of the American Astronomical Society (AAS). 


\section{MW Peer Stellar Halos Are Diverse, Reflecting a Diversity in Merger and Accretion Histories}

These observations show that MW peer stellar halos are diverse, spanning an order of magnitude or more in total mass and more than 1 dex in typical metallicity. This diversity is illustrated in Figure 2 (adapted from Figure 16 in [8]). Estimates of total stellar halo masses, metallicities at minor axis distances of $30 \mathrm{kpc}$, inferred 3D minor axis density gradients between 10 and $40 \mathrm{kpc}$, and minor axis metallicity gradients are shown in black for studies using resolved stars $[7,8,38,39,42-44]$. Gray symbols denote stellar halo masses or limits from integrated light studies $[36,45]$. Stellar halo masses have been extrapolated to give an estimate of total stellar halo masses. For this purpose, we use simulations of stellar halos including only accreted stars (e.g., [4,24,46]). For this particular analysis, we used the cosmologically-motivated particle-tagging models of [4], although we found that we recovered similar results with a particle-tagging model with more diverse accretion histories [26] and the accreted particles from two very different hydrodynamical simulations, Auriga and Illustris $[9,10,24,31,47]$. In these model accreted halos, most of the stellar halo mass lies at less than $10 \mathrm{kpc}$ galactocentric radius. The typical correction to estimate a total stellar halo mass from "aperture" measurements (at e.g., $10-40 \mathrm{kpc}$ ) is a factor of several [8], with an estimated accuracy of around $40 \%$ with little systematic dependence on stellar halo properties (see also $[9,10]$ ).

Panel a of Figure 2 shows that MW peers with a factor of 4 range in total stellar mass $\left(3 \times 10^{10} M_{\odot}\right.$ $<M_{*}<1.2 \times 10^{11} M_{\odot}$ ) show a factor of $\sim 20$ range in stellar halo fraction. We note that the correction of "aperture" stellar halo mass to a best estimate of total stellar halo mass is important, and brings the otherwise somewhat low "aperture" masses and limits presented by [36] into excellent agreement with $[8,45]$, and in accord with predictions from accretion-only models for stellar halo formation. Panels $\mathrm{c}$ and $\mathrm{d}$ show a range in power law density profile and metallicity gradients, respectively, with little clear correlation of either with stellar halo mass (or other quantities, as discussed in [8]). Importantly, panel $\mathrm{b}$ shows that stellar halo metallicity (as measured on the minor axis at $30 \mathrm{kpc}$ distance) and total stellar halo mass appear to correlate strongly-in agreement with the expectations of models of stellar halo formation through accretion only (as foreshadowed by [48] and explored by e.g., $[10,23,49]) — a$ result only accessible using resolved stellar populations.

This observational characterization of halos and confirmation of the predicted stellar halo metallicity-mass relation is important for a number of reasons.

- Following Section 2, it strongly suggests that the observations have indeed quantified the accreted stellar halo of galaxies.

- It shows that MW peers have a wide range of merger histories, where the Milky Way's low-mass stellar halo is very unusual. This is important because it tells us that the intuition that we have built about stellar halos from study of the Milky Way is incomplete.

- These accreted stellar halos can be used as a tool to quantify the properties of the most massive merger to have affected galaxies. The astronomical community now has quantitative access to the most massive event in individual galaxy's merger and accretion history.

The first and last of these ideas deserve some discussion.

We discuss the first point quantitatively in Figure 3 (adapted from Figure 1 of [9]), where we compare the observed stellar halo metallicity-mass relation with a set of modeled stellar halos in $10^{12} M_{\odot}$ dark matter halos from ([23], squares). The free parameters in these models are set only by the halo mass-stellar mass relation and metallicity-stellar mass relations of galaxies. When input model galaxies are realistic, the output accreted stellar halos closely reproduce the stellar halo metallicity-mass relation with appropriate normalization, slope, and scatter (see also [10] for an independent analysis using the Illustris hydrodynamical models with very similar results).

The relationship between stellar halo properties and the most massive accreted satellite have been articulated by $[9,10,23]$. In Figure 3, the models of [23] are color-coded by the mass-weighted mean stellar mass of all of the contributing satellites to the halo (termed "typical" satellite mass hereafter). The "typical" satellite mass is a strong function of the stellar halo mass (and metallicity). We provide 
an approximate mapping on the ordinate in blue in Figure 3. We also give an approximate merger ratio assuming a main galaxy mass at the time of merger of $\sim 3 \times 10^{10} M_{\odot}$ in green. Figure 3 suggests that one can broadly infer the masses of the largest satellites that were accreted by or merged into our nearby neighbors.

\section{Bulges and Central Black Holes in Disk-Dominated Galaxies Correlate Poorly with Merger History}

With a quantitative estimate of the most massive merger to have affected a galaxy in hand, we can now explore for the first time how MW peer merger history correlates with bulge and $\mathrm{BH}$ prominence. Figure 4 (Figure 2 of [9]) shows the bulge mass (left) and BH mass (right) as a function of stellar halo mass ( $\sim$ mass of the most massive merger/accretion partner). No significant correlations between bulge/BH masses and stellar halo masses are detected in this dataset. Galaxies with $M_{\text {stellar halo,tot }}>10^{9} M_{\odot}$ have an order of magnitude spread in B/T ratio or bulge mass and two orders of magnitude spread in $\mathrm{BH}$ mass.
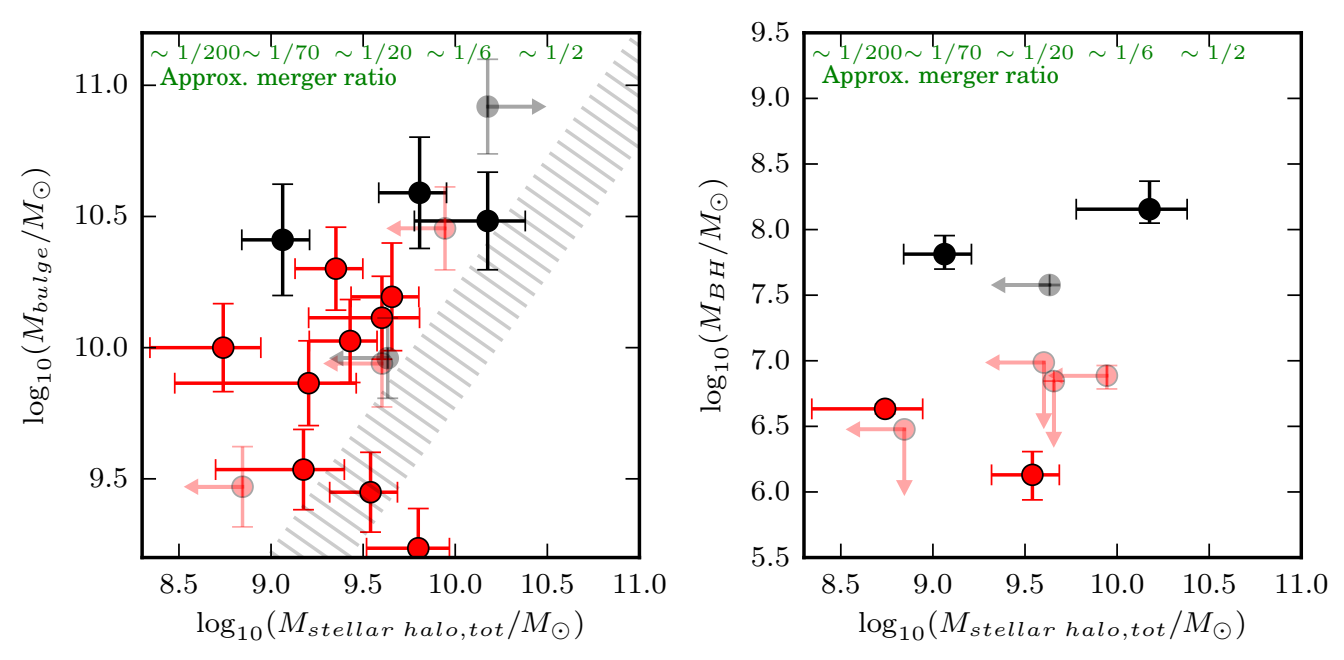

Figure 4. Bulge mass (left) and $\mathrm{BH}$ mass (right) as a function of stellar halo mass. Red denotes galaxies with low-mass "pseudobulges", black shows galaxies with higher-mass "classical" bulges; observational limits are shown with lighter shading. As argued in Figure 3, stellar halo mass reflects merger history, and approximate merger ratios are given in green. The shaded area in the left panel schematically illustrates what would be expected if there were a 1:1 correlation between stellar halo mass and bulge mass, as broadly expected in some simple modeling contexts (e.g., [1]). Figure 2 from [9], reproduced with permission of the AAS.

"Classical" bulges (black and grey) could have been expected to have been formed in major or minor mergers (e.g., [15]). Three galaxies with classical bulges (M31, NGC 3115, Cen $A^{1}$ ) indeed have massive metal-rich stellar halos-carrying $>20 \%$ of the total galaxy stellar mass-indicative of a minor or major merger. Yet, another three galaxies with classical bulges (M81, NGC 4258, NGC 7814) have less-massive stellar halos. Most notable among these is M81, with a large classical bulge and an anemic stellar halo containing only $2 \pm 0.9 \%$ of its total stellar mass. M81 shows no sign of any significant past major or minor merging activity that was expected to drive the formation of its classical bulge. A naïve one-to-one association between bulge and $\mathrm{BH}$ prominence and merging is ruled out, and places important constraints on models of bulge and BH formation and growth. In particular, such

1 While Cen A's minor axis metallicity at $30 \mathrm{kpc}$ suggests that it has a large stellar halo mass, no published estimate exists, and so it is not shown in Figure 4. 
systems may be an excellent testing ground for models in which bulge and $\mathrm{BH}$ growth is driven by other merger-independent processes.

\section{Conclusions}

A central goal of observational astrophysics is to measure the merger history of individual galaxies. Models predict that stellar halos provide such a measurement and allow us to explore how merger history—and in particular the most massive merger—affects galaxy properties.

Hubble Space Telescope and ground-based observations resolve individual stars in diffuse halos along the minor axes of nearby galaxies with total stellar masses similar to the Milky Way. The mass and metallicity of these stellar halos show a considerable range and correlate with each other, agreeing with models where they are formed by the disruption of dwarf galaxies alone. These models predict that stellar halo mass and metallicity constrain the mass of the largest accreted satellite galaxy. Consequently, the range in stellar halo mass and metallicity implies a range in the mass of the largest merged/accreted satellite galaxy. Intriguingly, in having a low mass and metallicity stellar halo, the Milky Way is not normal and likely experienced an unusually quiet merger history.

Motivated by models in which bulges and their central black holes (BHs) form or grow through galaxy merging, we then explore the relationship between these features and merger history. Bulge and central BH mass correlate poorly with stellar halo mass and therefore merger history. While half of the galaxies with the largest bulges and BHs have large stellar halos and thus have experienced a major or minor merger, half have small stellar halos and have never experienced a significant merger event. These systems may be an excellent testing ground for models where bulge and $\mathrm{BH}$ growth are driven by merger-independent processes.

Acknowledgments: This work benefited from the support from NSF grants AST 1008342 and AST 1514835 and HST grants GO-11613, GO-12213 and GO-13696 provided by NASA through a grant from the Space Telescope Science Institute, which is operated by the Association of Universities for Research in Astronomy, Inc., under NASA contract NAS5-26555. We appreciate the useful feedback from the two referees, and helpful conversations and insights from Sarah Loebman, Monica Valluri, Kohei Hattori, Ian Roederer, Adam Smercina, Bryan Terrazas, Denija Crnojevic, Duncan Forbes, Andrei Kravtsov, Nicolas Martin, and Oleg Gnedin.

Author Contributions: D.J.R.-S., R.S.dJ. and A.M. led the data reduction and photometry; B.H., A.M., E.F.B. and R.D'S. led the analysis; E.F.B. wrote the paper with contributions from all coauthors.

Conflicts of Interest: The authors declare no conflict of interest.

\section{References}

1. Hopkins, P.F.; Bundy, K.; Croton, D.; Hernquist, L.; Keres, D.; Khochfar, S.; Stewart, K.; Wetzel, A.; Younger, J.D. Mergers and Bulge Formation in $\Lambda$ CDM: Which Mergers Matter? Astrophys. J. 2010, 715, 202-229. [arXiv:astro-ph.CO/0906.5357].

2. Somerville, R.S.; Davé, R. Physical Models of Galaxy Formation in a Cosmological Framework. Annu. Rev. Astron. Astrophys. 2015, 53, 51-113. [1412.2712].

3. Bullock, J.S.; Kravtsov, A.V.; Weinberg, D.H. Hierarchical Galaxy Formation and Substructure in the Galaxy's Stellar Halo. Astrophys. J. 2001, 548, 33-46. [arXiv:astro-ph/0007295].

4. Bullock, J.S.; Johnston, K.V. Tracing Galaxy Formation with Stellar Halos. I. Methods. Astrophys. J. 2005, 635, 931-949. [arXiv:astro-ph/0506467].

5. Purcell, C.W.; Bullock, J.S.; Zentner, A.R. Shredded Galaxies as the Source of Diffuse Intrahalo Light on Varying Scales. Astrophys. J. 2007, 666, 20-33. [astro-ph/0703004].

6. Cooper, A.P.; Cole, S.; Frenk, C.S.; White, S.D.M.; Helly, J.; Benson, A.J.; De Lucia, G.; Helmi, A.; Jenkins, A.; Navarro, J.F.; et al. Galactic stellar haloes in the CDM model. Mon. Not. R. Astron. Soc. 2010, 406, 744-766. [arXiv:astro-ph.GA/0910.3211].

7. Monachesi, A.; Bell, E.F.; Radburn-Smith, D.J.; Bailin, J.; de Jong, R.S.; Holwerda, B.; Streich, D.; Silverstein, G. The GHOSTS survey - II. The diversity of halo colour and metallicity profiles of massive disc galaxies. Mon. Not. R. Astron. Soc. 2016, 457, 1419-1446. [1507.06657]. 
8. Harmsen, B.; Monachesi, A.; Bell, E.F.; de Jong, R.S.; Bailin, J.; Radburn-Smith, D.J.; Holwerda, B.W. Diverse stellar haloes in nearby Milky Way mass disc galaxies. Mon. Not. R. Astron. Soc. 2017, 466, 1491-1512. [1611.05448].

9. Bell, E.F.; Monachesi, A.; Harmsen, B.; de Jong, R.S.; Bailin, J.; Radburn-Smith, D.J.; D'Souza, R.; Holwerda, B.W. Galaxies Grow Their Bulges and Black Holes in Diverse Ways. Astrophys. J. 2017, 837, L8. [1702.06116].

10. D'Souza, R.; Bell, E. Accreted Metallicity-Stellar Mass Relationship. arXiv 2017, arXiv:1705.08442. [1705.08442].

11. Papovich, C.; Labbé, I.; Quadri, R.; Tilvi, V.; Behroozi, P.; Bell, E.F.; Glazebrook, K.; Spitler, L.; Straatman, C.M.S.; Tran, K.V.; et al. ZFOURGE/CANDELS: On the Evolution of M* Galaxy Progenitors from $\mathrm{z}=3$ to 0.5. Astrophys. J. 2015, 803, 26. [1412.3806].

12. Blanton, M.R.; Moustakas, J. Physical Properties and Environments of Nearby Galaxies. Annu. Rev. Astron. Astrophys. 2009, 47, 159-210. [0908.3017].

13. Schweizer, F.; Seitzer, P. Correlations between UBV colors and fine structure in E and S0 galaxies-A first attempt at dating ancient merger events. Astron. J. 1992, 104, 1039-1067.

14. Rothberg, B.; Joseph, R.D. A Survey of Merger Remnants. II. The Emerging Kinematic and Photometric Correlations. Astron. J. 2006, 131, 185-207. [astro-ph/0510019].

15. Kormendy, J.; Kennicutt, R.C., Jr. Secular Evolution and the Formation of Pseudobulges in Disk Galaxies. Annu. Rev. Astron. Astrophys. 2004, 42, 603-683. [astro-ph/0407343].

16. Johansson, P.H.; Naab, T.; Ostriker, J.P. Forming Early-type Galaxies in $\Lambda$ CDM Simulations. I. Assembly Histories. Astrophys. J. 2012, 754, 115. [1202.3441].

17. Ceverino, D.; Dekel, A.; Tweed, D.; Primack, J. Early formation of massive, compact, spheroidal galaxies with classical profiles by violent disc instability or mergers. Mon. Not. R. Astron. Soc. 2015, 447, 3291-3310. [1409.2622].

18. Sales, L.V.; Navarro, J.F.; Theuns, T.; Schaye, J.; White, S.D.M.; Frenk, C.S.; Crain, R.A.; Dalla Vecchia, C. The origin of discs and spheroids in simulated galaxies. Mon. Not. R. Astron. Soc. 2012, 423, 1544-1555. [arXiv:astro-ph.CO/1112.2220].

19. Terrazas, B.A.; Bell, E.F.; Henriques, B.M.B.; White, S.D.M.; Cattaneo, A.; Woo, J. Quiescence Correlates Strongly with Directly Measured Black Hole Mass in Central Galaxies. Astrophys. J. Lett. 2016, 830, L12. [1609.07141].

20. Terrazas, B.A.; Bell, E.F.; Woo, J.; Henriques, B.M.B. Supermassive Black Holes as the Regulators of Star Formation in Central Galaxies. Astrophys. J. 2017, 844, 170.

21. Koss, M.; Mushotzky, R.; Veilleux, S.; Winter, L.M.; Baumgartner, W.; Tueller, J.; Gehrels, N.; Valencic, L. Host Galaxy Properties of the Swift Bat Ultra Hard X-Ray Selected Active Galactic Nucleus. Astrophys. J. 2011, 739, 57. [arXiv:astro-ph.CO/1107.1237].

22. Ellison, S.L.; Patton, D.R.; Mendel, J.T.; Scudder, J.M. Galaxy pairs in the Sloan Digital Sky Survey - IV. Interactions trigger active galactic nuclei. Mon. Not. R. Astron. Soc. 2011, 418, 2043-2053. [1108.2711].

23. Deason, A.J.; Mao, Y.Y.; Wechsler, R.H. The Eating Habits of Milky Way-mass Halos: Destroyed Dwarf Satellites and the Metallicity Distribution of Accreted Stars. Astrophys. J. 2016, 821, 5. [1601.07905].

24. Vogelsberger, M.; Genel, S.; Springel, V.; Torrey, P.; Sijacki, D.; Xu, D.; Snyder, G.; Nelson, D.; Hernquist, L. Introducing the Illustris Project: simulating the coevolution of dark and visible matter in the Universe. Mon. Not. R. Astron. Soc. 2014, 444, 1518-1547. [1405.2921].

25. Purcell, C.W.; Bullock, J.S.; Zentner, A.R. The metallicity of diffuse intrahalo light. Mon. Not. R. Astron. Soc. 2008, 391, 550-558. [0805.2965].

26. Cooper, A.P.; D’Souza, R.; Kauffmann, G.; Wang, J.; Boylan-Kolchin, M.; Guo, Q.; Frenk, C.S.; White, S.D.M. Galactic accretion and the outer structure of galaxies in the CDM model. Mon. Not. R. Astron. Soc. 2013, 434, 3348-3367. [1303.6283].

27. Kirby, E.N.; Cohen, J.G.; Guhathakurta, P.; Cheng, L.; Bullock, J.S.; Gallazzi, A. The Universal Stellar Mass-Stellar Metallicity Relation for Dwarf Galaxies. Astrophys. J. 2013, 779, 102.

28. Zolotov, A.; Willman, B.; Brooks, A.M.; Governato, F.; Brook, C.B.; Hogg, D.W.; Quinn, T.; Stinson, G. The Dual Origin of Stellar Halos. Astrophys. J. 2009, 702, 1058-1067. [arXiv:astro-ph.GA/0904.3333].

29. Cooper, A.P.; Parry, O.H.; Lowing, B.; Cole, S.; Frenk, C. Formation of in situ stellar haloes in Milky Way-mass galaxies. Mon. Not. R. Astron. Soc. 2015, 454, 3185-3199. [1501.04630]. 
30. Pillepich, A.; Vogelsberger, M.; Deason, A.; Rodriguez-Gomez, V.; Genel, S.; Nelson, D.; Torrey, P.; Sales, L.V.; Marinacci, F.; Springel, V.; et al. Halo mass and assembly history exposed in the faint outskirts: The stellar and dark matter haloes of Illustris galaxies. Mon. Not. R. Astron. Soc. 2014, 444, 237-249. [1406.1174].

31. Monachesi, A.; Gómez, F.A.; Grand, R.J.J.; Kauffmann, G.; Marinacci, F.; Pakmor, R.; Springel, V.; Frenk, C.S. On the stellar halo metallicity profile of Milky Way-like galaxies in the Auriga simulations. Mon. Not. R. Astron. Soc. 2016, 459, L46-L50. [1512.03064].

32. Malin, D.; Hadley, B. HI in Shell Galaxies and Other Merger Remnants. Publ. Astron. Soc. Aust. 1997, 14, 52-58.

33. Martínez-Delgado, D.; Gabany, R.J.; Crawford, K.; Zibetti, S.; Majewski, S.R.; Rix, H.W.; Fliri, J.; Carballo-Bello, J.A.; Bardalez-Gagliuffi, D.C.; Peñarrubia, J.; et al. Stellar Tidal Streams in Spiral Galaxies of the Local Volume: A Pilot Survey with Modest Aperture Telescopes. Astron. J. 2010, 140, 962-967. [1003.4860].

34. Duc, P.A.; Cuillandre, J.C.; Karabal, E.; Cappellari, M.; Alatalo, K.; Blitz, L.; Bournaud, F.; Bureau, M.; Crocker, A.F.; Davies, R.L.; et al. The ATLAS ${ }^{3 D}$ project - XXIX. The new look of early-type galaxies and surrounding fields disclosed by extremely deep optical images. Mon. Not. R. Astron. Soc. 2015, 446, 120-143. [1410.0981].

35. Trujillo, I.; Fliri, J. Beyond $31 \mathrm{mag} \operatorname{arcsec}^{-2}$ : The Frontier of Low Surface Brightness Imaging with the Largest Optical Telescopes. Astrophys. J. 2016, 823, 123. [1510.04696].

36. Merritt, A.; van Dokkum, P.; Abraham, R.; Zhang, J. The Dragonfly nearby Galaxies Survey. I. Substantial Variation in the Diffuse Stellar Halos around Spiral Galaxies. Astrophys. J. 2016, 830, 62. [1606.08847].

37. Radburn-Smith, D.J.; de Jong, R.S.; Seth, A.C.; Bailin, J.; Bell, E.F.; Brown, T.M.; Bullock, J.S.; Courteau, S.; Dalcanton, J.J.; Ferguson, H.C.; et al. The GHOSTS Survey. I. Hubble Space Telescope Advanced Camera for Surveys Data. Astrophys. J. 2011, 195, 18.

38. Ibata, R.A.; Lewis, G.F.; McConnachie, A.W.; Martin, N.F.; Irwin, M.J.; Ferguson, A.M.N.; Babul, A.; Bernard, E.J.; Chapman, S.C.; Collins, M.; et al. The Large-scale Structure of the Halo of the Andromeda Galaxy. I. Global Stellar Density, Morphology and Metallicity Properties. Astrophys. J. 2014, 780, 386-406. [1311.5888].

39. Gilbert, K.M.; Kalirai, J.S.; Guhathakurta, P.; Beaton, R.L.; Geha, M.C.; Kirby, E.N.; Majewski, S.R.; Patterson, R.J.; Tollerud, E.J.; Bullock, J.S.; et al. Global Properties of M31's Stellar Halo from the SPLASH Survey. II. Metallicity Profile. Astrophys. J. 2014, 796, 76. [1409.3843].

40. Rejkuba, M.; Harris, W.E.; Greggio, L.; Harris, G.L.H.; Jerjen, H.; Gonzalez, O.A. Tracing the Outer Halo in a

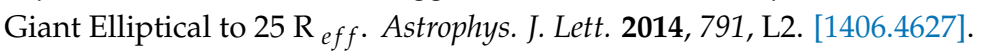

41. Peacock, M.B.; Strader, J.; Romanowsky, A.J.; Brodie, J.P. Detection of a Distinct Metal-poor Stellar Halo in the Early-type Galaxy NGC 3115. Astrophys. J. 2015, 800, 13. [1412.2752].

42. Bell, E.F.; Zucker, D.B.; Belokurov, V.; Sharma, S.; Johnston, K.V.; Bullock, J.S.; Hogg, D.W.; Jahnke, K.; de Jong, J.T.A.; Beers, T.C.; et al. The Accretion Origin of the Milky Way's Stellar Halo. Astrophys. J. 2008, 680, 295-311. [arXiv:0706.0004].

43. Xue, X.X.; Rix, H.W.; Ma, Z.; Morrison, H.; Bovy, J.; Sesar, B.; Janesh, W. The Radial Profile and Flattening of the Milky Way's Stellar Halo to 80 kpc from the SEGUE K-giant Survey. Astrophys. J. 2015, 809, 144. [1506.06144].

44. Sesar, B.; Jurić, M.; Ivezić, Ž. The Shape and Profile of the Milky Way Halo as Seen by the Canada-France-Hawaii Telescope Legacy Survey. Astrophys. J. 2011, 731, 4. [1011.4487].

45. D'Souza, R.; Kauffman, G.; Wang, J.; Vegetti, S. Parametrizing the stellar haloes of galaxies. Mon. Not. R. Astron. Soc. 2014, 443, 1433-1450. [1404.2123].

46. Rodriguez-Gomez, V.; Pillepich, A.; Sales, L.V.; Genel, S.; Vogelsberger, M.; Zhu, Q.; Wellons, S.; Nelson, D.; Torrey, P.; Springel, V.; et al. The stellar mass assembly of galaxies in the Illustris simulation: growth by mergers and the spatial distribution of accreted stars. Mon. Not. R. Astron. Soc. 2016, 458, 2371-2390. [1511.08804].

47. Grand, R.J.J.; Gómez, F.A.; Marinacci, F.; Pakmor, R.; Springel, V.; Campbell, D.J.R.; Frenk, C.S.; Jenkins, A.; White, S.D.M. The Auriga Project: the properties and formation mechanisms of disc galaxies across cosmic time. Mon. Not. R. Astron. Soc. 2017, 467, 179-207. [1610.01159]. 
48. Renda, A.; Gibson, B.K.; Mouhcine, M.; Ibata, R.A.; Kawata, D.; Flynn, C.; Brook, C.B. The stellar halo metallicity-luminosity relationship for spiral galaxies. Mon. Not. R. Astron. Soc. 2005, 363, L16-L20. [astro-ph/0507281].

49. Font, A.S.; Johnston, K.V.; Bullock, J.S.; Robertson, B.E. Phase-Space Distributions of Chemical Abundances in Milky Way-Type Galaxy Halos. Astrophys. J. 2006, 646, 886-898. [astro-ph/0512611].

(C) 2017 by the authors. Licensee MDPI, Basel, Switzerland. This article is an open access article distributed under the terms and conditions of the Creative Commons Attribution (CC BY) license (http:/ / creativecommons.org/licenses/by/4.0/). 